\title{
Very forward production and Feynman scaling of photons and neutrons at HERA
}

\author{
Hamlet Zohrabyan* (On behalf of the H1 Collaboration) \\ DESY,Hamburg/YerPhI,Yerevan \\ E-mail: zohrab@mail.desy.de
}

\begin{abstract}
Measurements of very forward neutron and photon production in deep-inelastic ep scattering at HERA as a function of Feynman-x are presented. The data are taken with the H1 detector in the years 2006 and 2007 and correspond to an integrated luminosity of $126 \mathrm{pb}^{-1}$. The analysis covers the range of negative four momentum transfer squared at the positron vertex $6<Q^{2}<100 \mathrm{GeV}^{2}$, inelasticity $0.05<y<0.6$ and the center-of-mass energy of the virtual photon-proton system $70<W<250 \mathrm{GeV}$. The dependence of the cross sections on $W$ is investigated. Predictions from DIS models and from models of the hadronic interactions of high energy Cosmic Rays are compared to the measurements.
\end{abstract}

Photon 2013,

20-24 May 2013

Paris, France

* Speaker. 


\section{Introduction}

Measurements of particle production at very small angles with respect to the proton beam direction (forward direction) in $e p$ collision are important for the understanding of proton fragmentation. Measurements of forward particle also provide important constraints for modeling of high energy air showers and thereby are very valuable for the understanding of high energy cosmic ray data.

New results from the H1 Experiment on very forward photon and neutron production in deepinelastic positron-proton scattering (DIS) are reported [1]. The photons and neutrons are measured in the Forward Neutron Calorimeter (FNC) [2,3], which was installed at $106 \mathrm{~m}$ from the $e p$ interaction point at a polar angel $0^{\circ}$ with respect to the proton beam direction at the interaction point. The acceptance of the FNC is defined by the aperture of the HERA beam-line magnets and is limited to scattering angles of $\theta<0.8 \mathrm{mrad}$ or pseudorapidity $\eta>7.9$.

\section{Forward Photon and Neutron Spectra in DIS}

Differential cross sections normalised to the inclusive DIS cross sections are measured for forward photons and neutrons as a function of the Feynman- $x$ variable $x_{F}=p_{\|}^{*} / p_{\| \text {max }}^{*}$ for three $W$ ranges: $70<W_{1}<130 \mathrm{GeV}, 130<W_{2}<190 \mathrm{GeV}$ and $190<W_{3}<250 \mathrm{GeV}$. The measurements are shown in Figures 1 - 6 together with the Monte Carlo (MC) model predictions.

The DJANGOH program [4] is used to generate inclusive DIS events. Higher order QCD effects are simulated using leading log parton showers as implemented in LEPTO [5], or using the Color Dipole Model (CDM) as implemented in ARIADNE [6]. In addition the RAPGAP [7] pionexchange model is used to describe the high $x_{F}$ part of forward neutron spectra. The data are also compared with Cosmic Ray (CR) hadronic interaction models commonly used for the simulation of CR air shower cascades: EPOS [8], QGSJET 01 [9] [10], QGSJET II [11] [12] and SIBYLL [13] [14].

In Figure 1 the cross sections measured for the most energetic photons with pseudorapidity $\eta>7.9$ is shown as a function of Feynman- $x$ variable for three $W$ region compared to DJANGOH (upper row) and CR (lower row) models. Both MC models, LEPTO and CDM, significantly overestimate the rate of forward photons by $\sim 70 \%$. The shape of the LEPTO prediction is close to the data, while CDM exhibits a harder $x_{F}$ behaviour. The CR models are closer to data in absolute values, but are also higher by $\sim 30-50 \%$. These properties are well visible in the MC/Data ratios depicted in Figure 2. The best description of the forward photon measurements is provided by QGSJET model. In Figure 3 the $W_{2} / W_{1}$ and $W_{3} / W_{1}$ ratios of the $x_{F}$ spectrum are shown for DJANGOH (upper row) and CR (lower row) models. The ratio for the data is consistent with unity within errors and being independent of of $x_{F}$, supporting the validity of Feynman scaling. The ratios for the standard DIS models (LEPTO and CDM) are slightly larger, and the ratios for CR models are lower than unity.

The results of the $x_{F}$ measurements of forward neutron production are presented in Figure 4. In the upper row a comparison of the data and the CDM, RAPGAP- $\pi$ (pion-exchange) and the combination of these models is shown. The 'combined' model describes the data well. The comparison of the data with the CR hadronic interaction models depicted in lower row of Figure 
4. In the Figure $5 \mathrm{MC}$ to Data Ratios is given for combination of CDM and RAPGAP- $\pi$ model and in the lower row for CR models. One can say the EPOS gives the best description of the data. In Figure 6 the $W_{2} / W_{1}$ and $W_{3} / W_{1}$ ratios of the $x_{F}$ spectra are presented for Combined (upper row) and the CR (lower row) models. The ratio for the data is consistent with unity and independent of $x_{F}$ within errors, supporting the validity of Feynman scaling. The combined model deviates from unity but does not depend on $x_{F}$ while most of the CR models show a dependence with $x_{F}$ indicating the $x_{F}$ scaling is broken in these models.

\section{Fraction of DIS events with forward photons and neutrons}

The measurement of forward photons and neutrons allows a test of the limiting fragmentation hypothesis, which implies that forward particle production is insensitive to $W$. To investigate this prediction, the ratio of the forward photon and neutron production cross section to the inclusive DIS cross section is measured as a function of $W$ and compared with the standard DIS and the CR models in Figure 7.

Within the uncertainties measured fraction of DIS events with photons and neutrons is independent from $W$, in agreement with the hypothesis of limiting fragmentation. LEPTO and CDM indicate a significant differences in normalization compared to data, but independence of $W$ for photons. The CR models indicate a large spread of their predictions and some $W$ dependencies for both, the photon and the neutron yields. For forward neutrons the combination of the standard DIS and pion exchange models describe the data well, but shows some $W$ dependencies.

\section{Summary}

The production of forward photons and neutrons has been studied in deep inelastic positron-proton scattering in the kinematic range $6<Q^{2}<100 \mathrm{GeV}^{2}, 0.05<y<0.6$ and in the pseudorapidity range of photons and neutrons $\eta>7.9$ in laboratory frame. The Monte Carlo models overestimate the production rate of photons. While LEPTO describes the shapes well, CDM predicts a harder $x_{F}$ spectrum. The CR models considered are closer to the data in normalisation. For forward neutrons none of the models describes the data well. The combination of standard fragmentation and the pion-exchange models describes the measured $x_{F}$ neutron spectra well.

The measurements support the limiting fragmentation hypothesis and are consistent with Feynman scaling. The present measurements provides further insight into the proton fragmentation process and can be used for models tuning.

\section{References}

[1] Forward photon spectra measured in FNC, H1prelim-13-012, http://www-h1.desy.de/psfiles/confpap/DIS2013/H1 prelim-13-012.pdf

[2] F.D.Aaron et al. Measurement of Photon Production in the Very Forward Direction in Deep-Inelastic Scattering at HERA, Eur.Phys.J. C71 (2011) 1771

[3] F.D.Aaron et al. Measurement of Leading Neutron Production in Deep-Inelastic Scattering at HERA, Eur.Phys.J. C68 (2010) 381-399 
[4] K.Charchula, G.A.Schuler and H.Spiesberger, DJANGOH 1.4, Comput.Phys.Commun. 81,381 (1994).

[5] G.Ingelman, A.Edin and J.Rathsman, LEPTO 6.5, Comput.Phys.Commun.101 (1997) 108 [hep-ph/9605286].

[6] L.Lonnblad, ARIADNE 4.10, Comput.Phys.Commun.71 (1992) 15.

[7] H. Jung, RAPGAP 3.1, Comp. Phys. Commun. 86 (1995) 147.

[8] K.Werner,F.-M.Liu and T.Pierog, Phys.Rev. C74 (2006) 044902 [hep-ph/0506232].

[9] N.N.Kalmykov and S.S.Ostapchenko, Phys.Atom.Nucl. 56 (1993) 346.

[10] N.N.Kalmykov, S.S.Ostapchenko, A.I.Pavlov, Nucl.Phys.Proc.Suppl.52B (1997) 17.

[11] S.S.Ostapchenko, Phys.Rev. D74 (2006) 014026 [hep-ph/0505259].

[12] S.S.Ostapchenko, AIP Conf.Proc.928 (2007) 118 [arXiv:0706.3784].

[13] J.Engel et al., Phys.Rev. D46 (1992) 5013.

[14] E.-J.Ahn et al., Phys.Rev. D80(2009)094003 [arXiv:0906.4113].
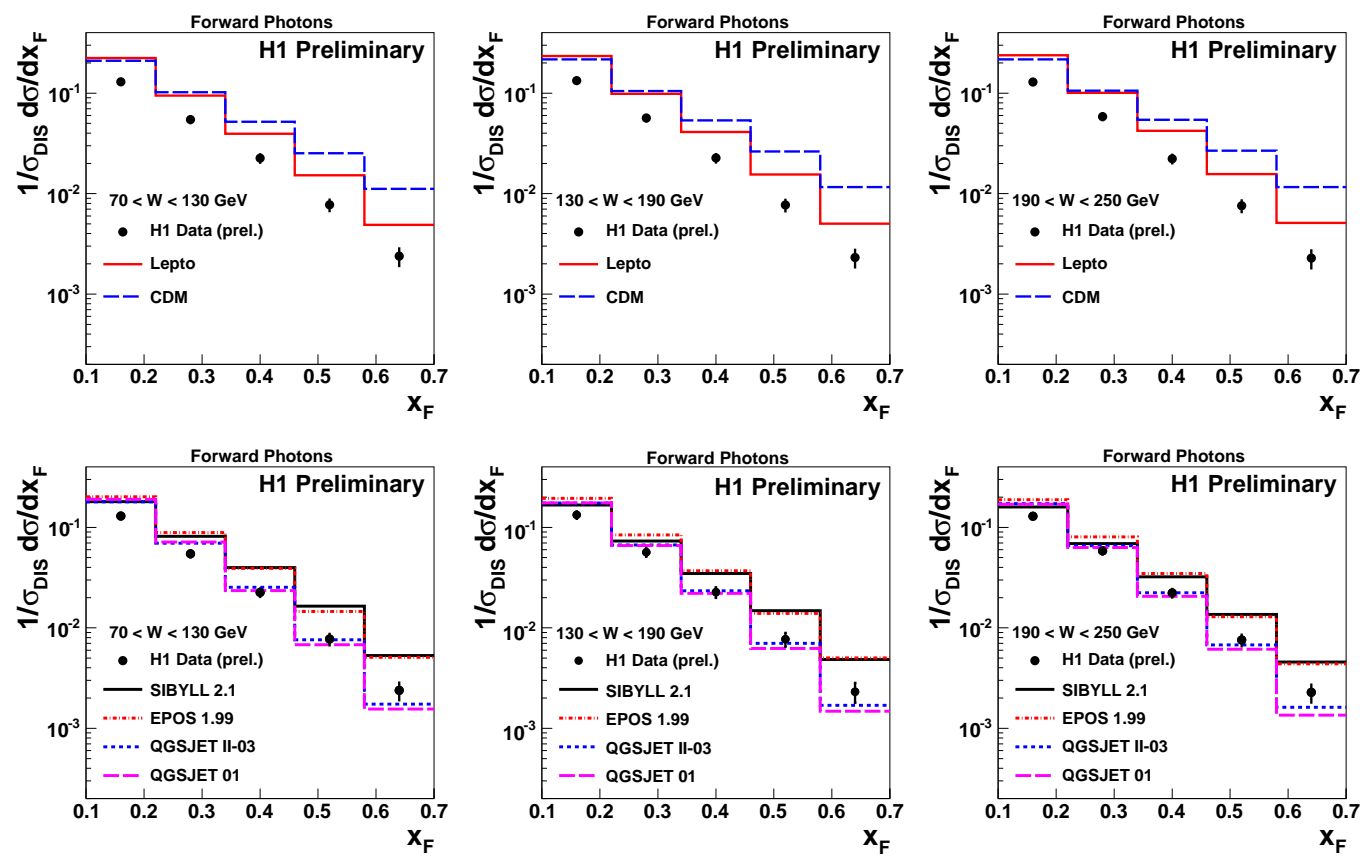

Figure 1: Normalized cross sections for forward photon production as a function of $x_{F}$ compared to predictions of the LEPTO and CDM MC models (upper row), and Cosmic Ray hadronic interaction models (lower row) for three $W$ ranges. 

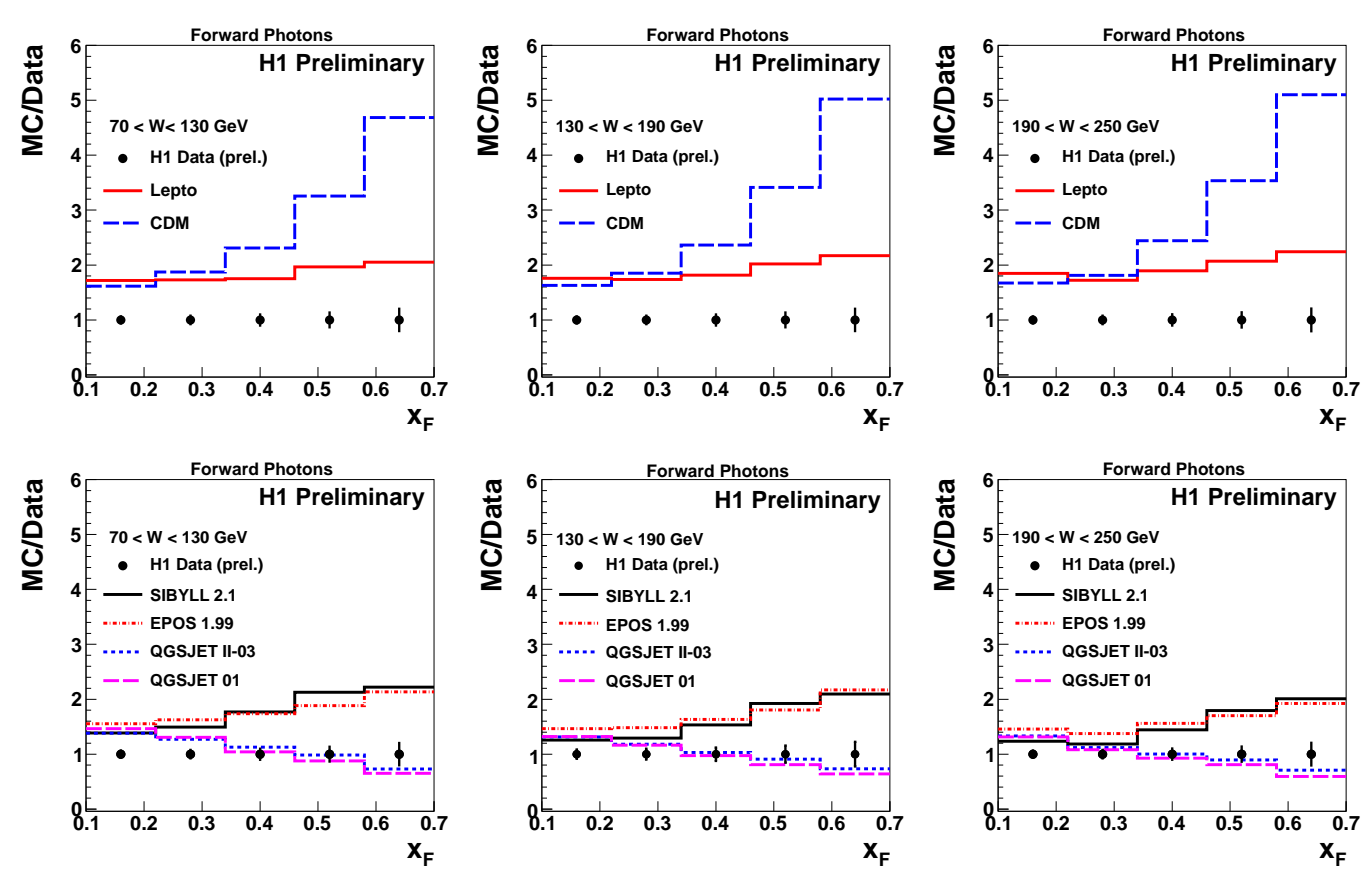

Figure 2: The ratios of MC predictions to the data as as a function of $x_{F}$ compared to predictions of the LEPTO and CDM MC models (upper row), and Cosmic Ray hadronic interaction models (lower row) for three $W$ ranges.
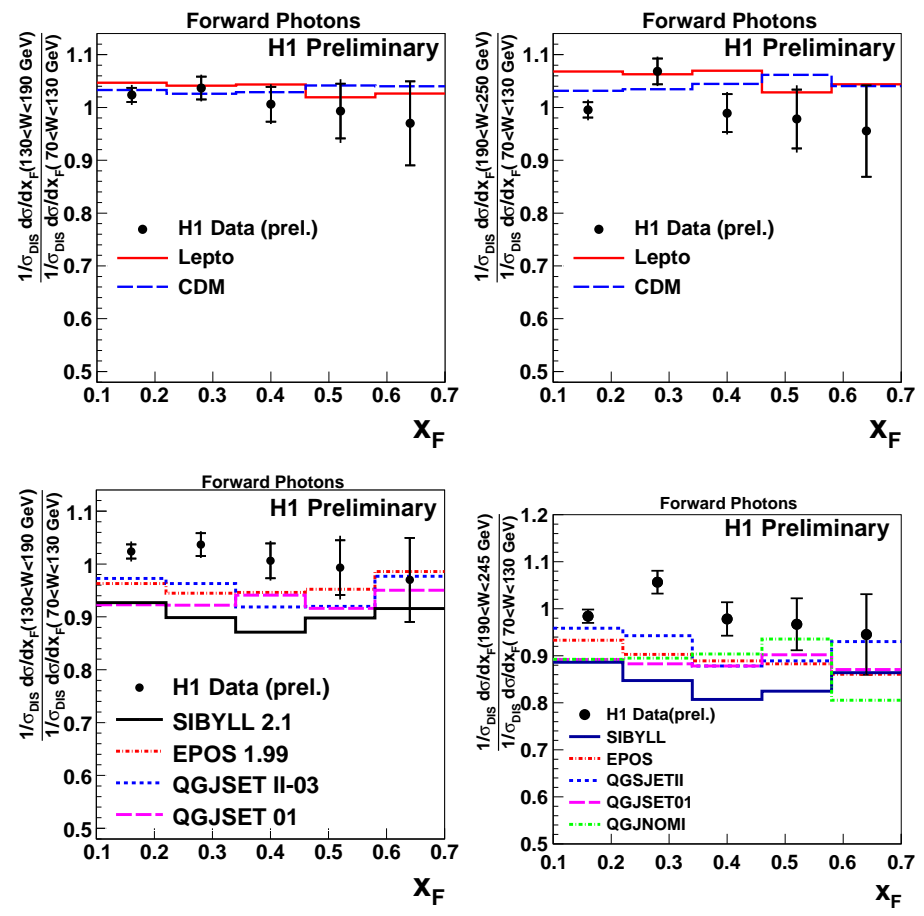

Figure 3: The Photons $W_{2} / W_{1}$ and $W_{3} / W_{1} x_{F}$ spectra ratios for LEPTO and CDM MC models (upper row), and Cosmic Ray hadronic interaction models (lower row). 

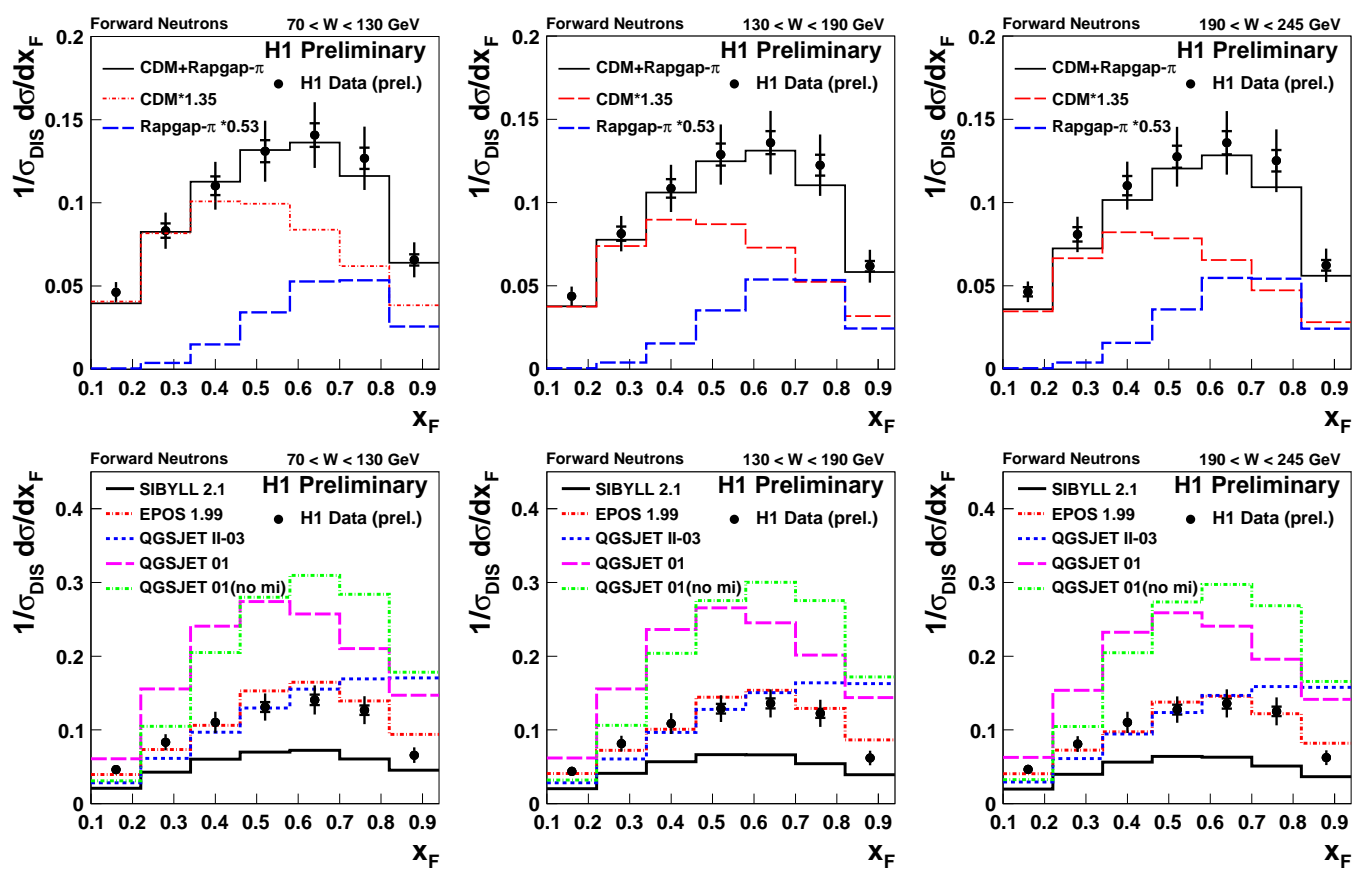

Figure 4: Normalized cross sections for forward neutron production as a function of $x_{F}$ compared to CDM, the RAPGAP pion-exchange model and their combination using scaling factors (upper row), and Cosmic Ray hadronic interaction models (lower row) for three $W$ ranges.
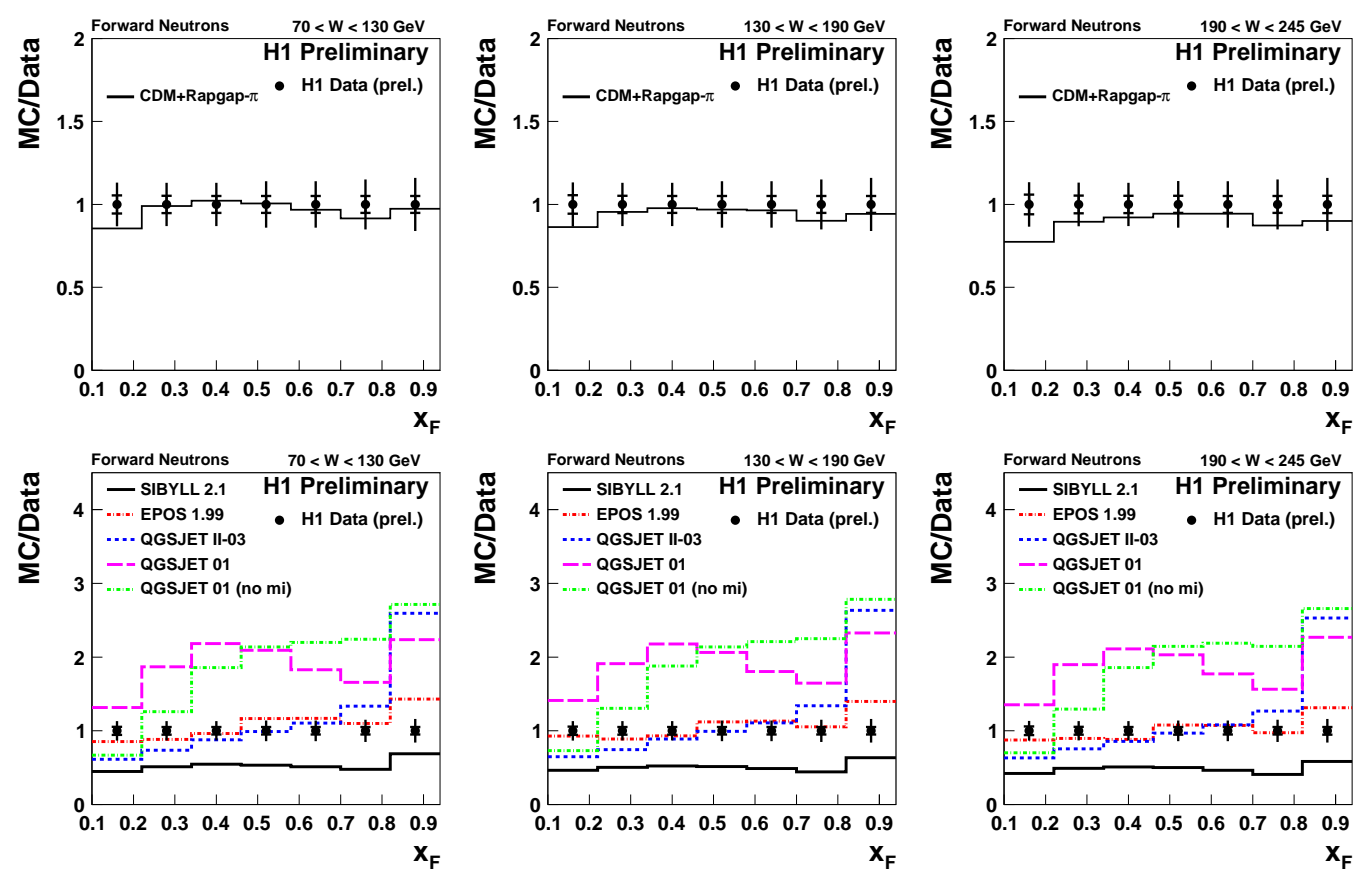

Figure 5: The ratios of MC predictions to the data as a function of $x_{F}$ compared to predictions of the Combined(CDM+RAPGAP) models (upper row), and Cosmic Ray hadronic interaction models (lower row) for three $W$ ranges. 

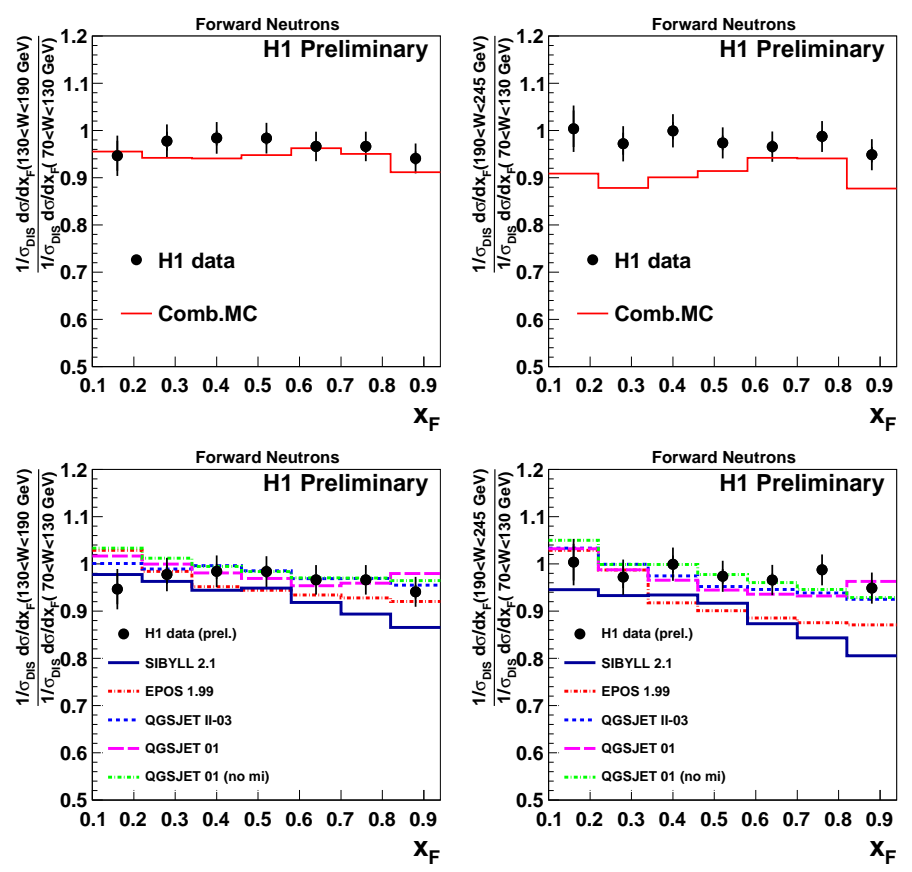

Figure 6: The Neutrons $W_{2} / W_{1}$ and $W_{3} / W_{1} x_{F}$ spectra ratios for Combined CDM and RAPGAP pionexchange models (upper row), and Cosmic Ray hadronic interaction models (lower row).
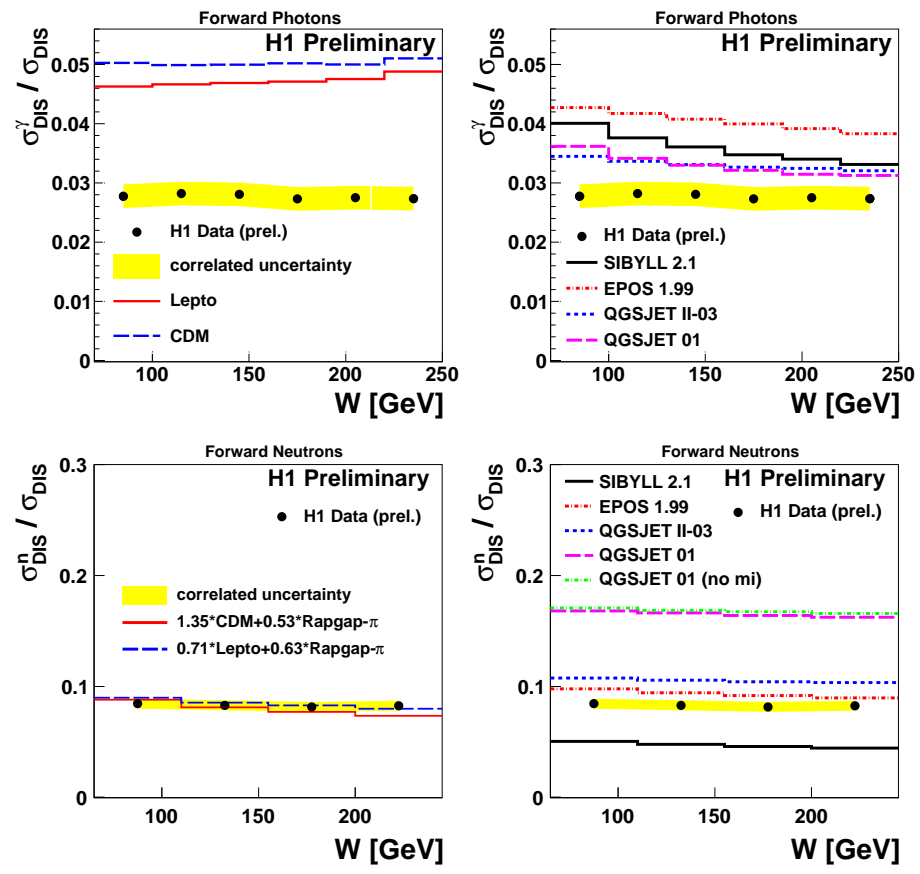

Figure 7: Fraction of DIS events with forward photons and neutrons as a function of $W$. Upper (lower) row shows the distributions for the forward photons(neutrons). The MC models are compared to the measurements. 